\title{
Stelis franconica BLÜTHGEN 1930 (Apoidea - Megachilidae - Anthidiini), une nouvelle espèce pour la faune de France et mise au point sur la liste des Stelis (PANZER 1806) de France
}

\author{
par David GENOUD* et Éric DUFRÊNE**
}

\begin{abstract}
Résumé. En l'espace de deux années, Stelis franconica BLÜTHGEN 1930 a été découverte dans trois départements français différents. La première découverte dans les Pyrénées est particulièrement surprenante compte tenu de la distribution antérieurement connue de l'espèce. L'absence d'Osmia mustelina GERSTAECKER 1869 en France et l'abondance d'Osmia emarginata LEPELETIER 1841 sur la station pyrénéenne aux mêmes dates amènent à considérer cette dernière espèce comme l'hôte probable.
\end{abstract}

\begin{abstract}
Within two years, Stelis franconica BLÜTHGEN 1930 was discovered in three different French departments. The first specimen was discovered in Pyrenees which is particularly surprising considering the previously known distribution of the species. The absence of Osmia mustelina GERSTAECKER 1869 in France and the abundance of Osmia emarginata LEPELETIER 1841 on the Pyrenean site for the same dates lead us to consider the latter species as the likely host.
\end{abstract}

Mots-clés : Stelis franconica; Osmia emarginata ; France.

Key-words : Stelis franconica; Osmia emarginata ; France.

La faune du Paléarctique comprend 28 espèces connues du genre Stelis réparties en 5 sousgenres (dont deux espèces au sous-genre incertain ${ }^{1}$ ). RASMONT et al. (1995) établissaient une liste de 9 espèces pour la faune de France. Toutefois cette liste n'est plus à jour puisque Stelis odontopyga NoskiewICZ 1925 fait partie intégrante de la faune de France. En effet, plusieurs collecteurs du réseau Apoidea Gallica

* Observatoire des Abeilles (OA), 2 Domaine Bellevue, 11290 Arzens - dge-davidgenoud@orange.fr

** Observatoire des Abeilles (OA), 9 allée des pins, 91470

Forges-les-Bains - eric.dfn@wanadoo.fr ont pu faire valider cette espèce dans leur collection (Gironde - 33, $\mathrm{DG}^{2}$; Manche - 50, ED). Il est probable que d'autres données de cette espèce existent car Osmia spinulosa KIRBY 1802, est largement répartie en France, or cette osmie hélicicole est l'hôte connu de $S$. odontopyga (AMIET, 2004).

Ce premier ajout portait la faune des Stelis de France à 10 espèces. Cette nouvelle liste pouvait paraître conforme à la diversité taxonomique attendue pour notre pays. Seules deux espèces parmi celles présentes dans les pays limitrophes, Stelis minima LEPELETIER \& SERVILLE 1825 et

\footnotetext{
${ }^{1}$ Stelis ortizi SCHWARZ \& GUSENLEITENER 2010 ; espèce ibérique et Stelis rhodia MAVROMOUSTAKIS 1960 ; taxon cypro-turque

${ }^{2}$ Afin d'alléger le texte les récolteurs sont cités comme suit : David Genoud (DG) ; Eric Dufrêne (ED) ;

Gérard Le Goff (GLG) ; Vincent Lefebvre (VL).
} 
Stelis franconica BLÜTHGEN 1930, ne sont pas connues de France (ni de la péninsule ibérique). $S$. franconica est considérée comme éteinte en Allemagne (http://westpalbees.myspecies.info/ taxonomy \& http://maps.iucnredlist.org/map). Toutefois, l'espèce est connue de plusieurs vallées alpines suisses aux confins de la frontière française. (AMIET, 2004 ; http://lepus.unine.ch/ carto) et restait donc potentiellement présente sur l'ensemble de l'arc alpin.

En 2014, un inventaire ciblé sur la diversité de la mélittofaune de la Réserve Naturelle Nationale de la Massane (Pyrénées-Orientales - 66) a permis la capture, aux coupelles colorées, d'une femelle de Stelis franconica (RN de la Massane DG). Son hôte, O. mustelina GERSTAECKER 1869 est présent en Allemagne et en Suisse (SCHEUCHL, 1996 ; AMIET, 2004) mais n'est pas connu de France. Il existe une espèce très proche en France, Osmia emarginata LEPELETIER 1841 (MüLLER, 2016), longtemps confondue avec la précédente. $O$. emarginata est abondante sur le site où elle colonise les fissures rocheuses de part et d'autre de la crête des Albères donc tant du côté français qu'ibérique. O. emarginata pourrait être un nouvel hôte pour S. franconica. La batterie de coupelles était située en France à quelques mètres de la frontière. Compte tenu de la proximité immédiate de l'Espagne, l'aire de répartition des deux espèces chevauche très probablement les deux pays. Notons que $S$. franconica n'est pas signalée en Espagne dans la littérature (ORTIZ-SANCHEZ, 2011 ; ORNOSA et al. 2008).

La même année un mâle de Stelis franconica a été collecté le 6 juin 2014 à Jausiers dans le département des Alpes-de-Haute-Provence (04) dans le Parc National du Mercantour (VL MNHN).

Enfin, une femelle de cette espèce a également été capturée le 23 juin 2015 sur la commune de Val-Maravel dans le département de la Drôme (26) lors d'une prospection commune de quelques membres de l'Observatoire des Abeilles (ED, GLG).

L'ensemble des données a été vérifié par les collecteurs et plusieurs déterminateurs (Eric Dufrêne, Gérard Le Goff, Matthieu Aubert).

L'espèce a été capturée dans une gamme d'altitudes allant de $980 \mathrm{~m}$ (sous le Puig de Sallfort - RN de la Massane) à 1800 m
(Mercantour) et l'espèce est connue en Suisse jusqu'à 2000 m (AMIET, 2004).

Sa présence dans les Pyrénées n'était pas la plus attendue du fait de son absence d'Espagne et des altitudes assez modestes sur le massif environnant. Par contre il est assez logique qu'on la retrouve aux étages montagnards et alpins du massif des Alpes, comme déjà observée en Suisse et il est probable qu'à l'avenir de nouvelles stations soient découvertes.

La faune de France comprend donc maintenant 11 espèces de Stelis, répertoriées ci-dessous :

- Stelis (Heterostelis) annulata LEPELETIER 1841

- Stelis (Protostelis) signata LATREILLE 1809

- Stelis (Pseudostelis) minuta LEPELETIER \& AUDINET-SERVILLE 1825

- Stelis (Stelidomorpha) nasuta LATREILLE 1809

- Stelis (Stelis) breviuscula NYLANDER 1848

- Stelis (Stelis) franconica BLÜTHGEN 1930

- Stelis (Stelis) odontopyga NoskiEWICZ 1926

- Stelis (Stelis) ornatula KLUG 1807

- Stelis (Stelis) phaeoptera KIRBY 1802

- Stelis (Stelis) punctulatissima KIRBY 1802

- Stelis (Stelis) simillima MORAWIT 1876

\section{Remerciements}

Nos remerciements vont à Gérard Le Goff, Géraud de Prémorel, Matthieu Aubert pour leurs prêts de matériels, leurs relectures et les nombreux échanges relatifs à la connaissance générale des abeilles, à Vincent Lefebvre (MNHN) qui nous a aimablement autorisé à diffuser et publier sa donnée, à Christophe PRAZ pour l'apport bibliographique et les traductions de certaines publications. Enfin au personnel de la Réserve Naturelle de la Massane (Joseph Garrigue, Jean-André Magdalou, Diane Sorel) pour tout leur travail et leur disponibilité.

\section{Bibliographie}

Amiet F, Herrmann M, Müller A \& NEUMEYer R, 2004. Fauna Helvetica 9 : Apidae 4 : Anthidium, Chelostoma, Coelioxys, Dioxys, Heriades, Lithurgus, Megachile, Osmia, Stelis. 
Centre Suisse de Cartographie de la Faune \& Schweizerische Entomologische Gesellschaft, Neuchâtel, 273 p.

BANASZAK J \& ROMASENKO L, 2001. Megachilid bees of Europe. Bydgoszcz University of Kazimierz Wielki eds., Bydgoszcz, Polska.

GogalA A, 2014. Megachilid bees of Slovenia (Hymenoptera: Apoidea: Megachilidae). SCOPOLIA, 80: 1-195.

Kuhlmann M et al., 2016. Checklist of the Western Palaearctic Bees (Hymenoptera: Apoidea: Anthophila). http:// westpalbees.myspecies.info

Michener CD, 2007. The bees of the world. John Hopkins University Press, Baltimore, Mariland. $953 \mathrm{p}$.

MÜLler A, 2016. - Palaearctic Osmiine Bees, ETH Zürich, http://blogs.ethz.ch/osmiini

ORTIZ-SANCHEZ F-J, 2011. Lista actualizada de las especies de abejas de Espana (Hymenoptera: Apoidea: Apiformes). Bol. De la Soc. Entomol. Aragonesa (SEA), 49: 265-281.

Ornosa C, OrTiz-SANChez F-J, TORRes F, 2008. Catalogo de los Megachilidae del mediterranea occcidental (Hymenoptera, Apoidea) III. Anthidini y Dioxini. Graellsia, 64(1): 61-86.

SCHEUCHL E, 1996 . Illustrierte Bestimmungsschlüssel der Wildbienen Deutschlands und Österreichs. Band II: Megachilidae - Melittidae. Velden, $116 \mathrm{p}$.

Westrich P, 1989. Die wildbienen BadenWürtembergs. Eugen Ulmer, Stuttgart, Germany. 972 p. 2 volumes. 